\title{
Divergence of a conserved elongation factor and transcription regulation in budding and fission yeast
}

\author{
Gregory T. Booth, ${ }^{1}$ Isabel X. Wang, ${ }^{2}$ Vivian G. Cheung, ${ }^{2}$ and John T. Lis ${ }^{1}$ \\ ${ }^{1}$ Department of Molecular Biology and Genetics, Cornell University, Ithaca, New York 14853-2703, USA; ${ }^{2}$ Life Sciences Institute, \\ University of Michigan, Ann Arbor, Michigan 48109, USA
}

\begin{abstract}
Complex regulation of gene expression in mammals has evolved from simpler eukaryotic systems, yet the mechanistic features of this evolution remain elusive. Here, we compared the transcriptional landscapes of the distantly related budding and fission yeast. We adapted the Precision Run-On sequencing (PRO-seq) approach to map the positions of RNA polymerase active sites genome-wide in Schizosaccharomyces pombe and Saccharomyces cerevisiae. Additionally, we mapped preferred sites of transcription initiation in each organism using PRO-cap. Unexpectedly, we identify a pause in early elongation, specific to $S$. pombe, that requires the conserved elongation factor subunit Spt4 and resembles promoter-proximal pausing in metazoans. PRO-seq profiles in strains lacking Spt4 reveal globally elevated levels of transcribing RNA Polymerase II (Pol II) within genes in both species. Messenger RNA abundance, however, does not reflect the increases in Pol II density, indicating a global reduction in elongation rate. Together, our results provide the first base-pair resolution map of transcription elongation in S. pombe and identify divergent roles for Spt4 in controlling elongation in budding and fission yeast.
\end{abstract}

[Supplemental material is available for this article.]

Budding and fission yeast are both widely used model organisms for the study of transcription, yet their genomes are as distantly related as metazoans and budding yeast (Dujon 2010). Details of transcription and its regulation have been characterized through a variety of high-resolution and genome-wide studies for Saccharomyces cerevisiae (Hahn and Young 2011; Pelechano and Steinmetz 2013). However, although location and production of genomic RNA has been investigated in fission yeast (Wilhelm et al. 2008; DeGennaro et al. 2013), various aspects of the transcriptional landscape of Schizosaccharomyces pombe remain more vague. Through our investigation of elongating RNA polymerase distributions in S. pombe, we unexpectedly found evidence of promoter-proximal pause-like distributions of RNA polymerase across the $S$. pombe genome. These findings prompted our inquiry into the mechanistic differences between budding and fission yeast at the level of transcription.

Unlike most well-studied metazoans, budding yeast displays a relatively uniform distribution of elongating Pol II across transcription units (Steinmetz et al. 2006). The lack of significant barriers to elongation in S. cerevisiae supports a model in which Pol II recruitment and initiation are the predominant steps regulating gene activity (Stargell and Struhl 1996; Ptashne and Gann 1997). In contrast, the majority of genes in metazoans, including mammals and flies, are intricately regulated at a promoter-proximal pausing step during elongation (Fuda et al. 2009; Adelman and Lis 2012). Characteristics of transcription elongation also differ between metazoans and S. cerevisiae beyond the cleavage and polyadenylation signal (CPS) (Porrua and Libri 2015). In contrast to budding yeast, elongating Pol II in mammals experiences post-CPS slowing or pausing while continuing to transcribe for several kilobases prior to termination (Proudfoot 1989; Gromak et al. 2006; Core et al. 2008; Laitem et al. 2015). These differences in Pol II dynamics reflect distinct mechanisms for controlling gene transcription

Corresponding author: johnlis@cornell.edu

Article published online before print. Article, supplemental material, and publication date are at http://www.genome.org/cgi/doi/10.1101/gr.204578.116. that likely reflect the vast evolutionary distance separating these organisms.

How promoter-proximal pausing evolved into a ubiquitous mechanism regulating transcription in mammals is unknown. Metazoans with pervasive pausing possess a four-subunit complex termed negative elongation factor (NELF) with no known orthologs in yeast, C. elegans, or plants (Hartzog and Fu 2013). NELF cooperates with DRB-sensitivity inducing factor (DSIF), composed of the highly conserved Spt 4 and Spt5 subunits, to induce stable pausing of RNA polymerase shortly after initiation (Wada et al. 1998; Wu et al. 2003). However, studies in which NELF was highly depleted in vivo have shown that cells still retain substantial promoter-proximal pausing (Brannan et al. 2012; Core et al. 2012), consistent with the idea that DSIF is sufficient to induce promoter-proximal pausing (Wada et al. 1998). In fact, recent studies found that, despite lacking NELF homologs, some C. elegans genes are paused under nutrient-limiting conditions (Kruesi et al. 2013; Maxwell et al. 2014). Nonetheless, it remains unknown whether such pausing in vivo is dependent on Spt4-Spt5.

Pause release in metazoans is modulated by the kinase activity of cyclin dependent kinase 9 (CDK9), a complex referred to as positive transcription elongation factor b (pTEFb) (Wada 1998). CDK9 phosphorylates NELF, DSIF, and Pol II to permit pause release and elongation into the gene body (Peterlin and Price 2006). This transition coincides with the release of NELF from the complex, at which point phosphorylated DSIF is converted into a positive elongation factor (Ping 2000). In all eukaryotes, DSIF stably associates with transcribing Pol II throughout the duration of transcription. Mutations in either DSIF subunit (Spt4-Spt5) in S. cerevisiae have been found to result in elongation defects (Swanson et al. 1991; Keogh et al. 2003), but how Spt4-Spt5 evolved an additional role in promoter-proximal pausing remains unknown.

(c) 2016 Booth et al. This article is distributed exclusively by Cold Spring Harbor Laboratory Press for the first six months after the full-issue publication date (see http://genome.cshlp.org/site/misc/terms.xhtml). After six months, it is available under a Creative Commons License (Attribution-NonCommercial 4.0 International), as described at http://creativecommons.org/licenses/by-nc/4.0/. 
Our understanding of pausing as well as numerous other transcriptioncoupled phenomena has been greatly enhanced through the use of nuclear-run on approaches (Love et al. 1985). More recently, Global and Precision Run-On sequencing (GRO/PRO-seq) have provided genome-wide views of the distribution of engaged RNA Polymerases with strand specificity in metazoan organisms (Core et al. 2008; Kwak et al. 2013). Despite the use of various run-on-based approaches in $S$. cerevisiae (Pelechano et al. 2010; Rodríguez-Gil et al. 2010; McKinlay et al. 2011; Jordán-Pla et al. 2014), neither GRO-seq nor PRO-seq has been fully demonstrated in yeast. Here, we use a yeast-optimized, Precision Run-On sequencing approach to assay elongating RNA polymerases genomewide in distantly related budding and fission yeast, providing the first base-pair resolution transcription analysis in $S$. pombe. We further apply this technique to investigate the importance of the Spt4-Spt5 complex in global transcription elongation. Comparing the effect of Spt 4 deletion in both $S$. cerevisiae and $S$. pombe provides insight into the evolution of its role in regulating Pol II transcription elongation in eukaryotes.

\section{Results}

PRO-cap and PRO-seq capture sites of transcription initiation and elongation in S. cerevisiae and S. pombe

We first set out to map positions of all elongation competent RNA polymerases in both budding and fission yeast using the Precision Run-On sequencing (PROseq) protocol, which we adapted for use in yeast. Furthermore, we used Precision Run-On 5' cap sequencing (PRO-cap), a modification to the PRO-seq protocol which includes selection for short, capped nascent RNAs, to precisely identify the initiation sites for transcription units in both species (Supplemental Table S2, S. cerevisiae; Supplemental Table S3, S. pombe; Kwak et al. 2013).

PRO-seq signal in $S$. pombe is observed across the transcription unit of active genes, providing the direction, relative amount and distribution of elongating polymerases (Fig. 1A, top track). In contrast, PRO-cap signal is largely confined to the $5^{\prime}$ regions near annotated TSSs, indicating a successful enrichment of nascent RNA 5' cap sites (Fig. 1A, lower track).

To more precisely define the initiation sites of transcription units, we identified the base with the most PRO-cap reads above background within 250 bases upstream of or downstream from the annotated TSS (Fig. 1A; Supplemental Fig. S1A, green models).

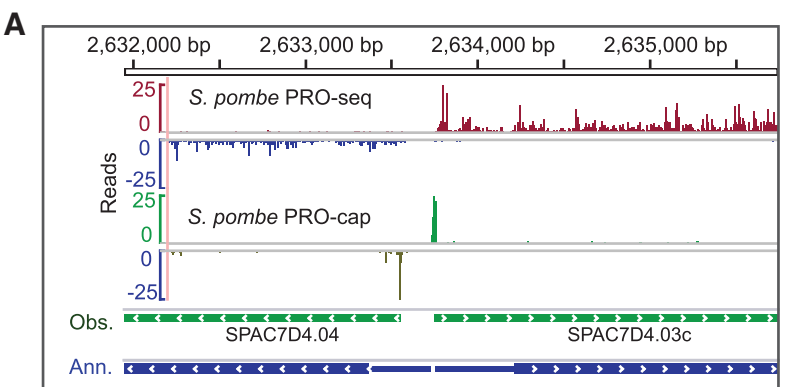

B

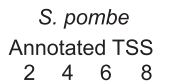

$\begin{array}{llllrl}0 & 2 & 4 & 6 & 8 & 10\end{array}$
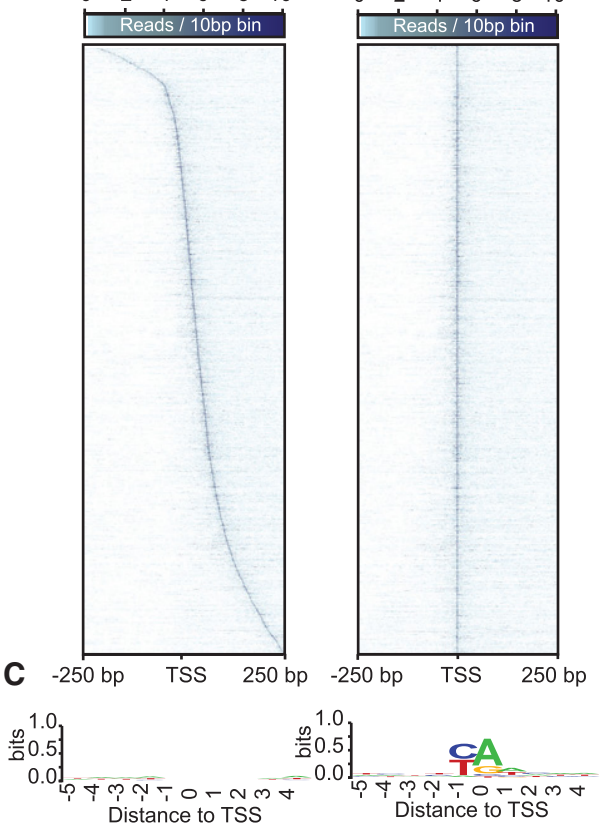

D

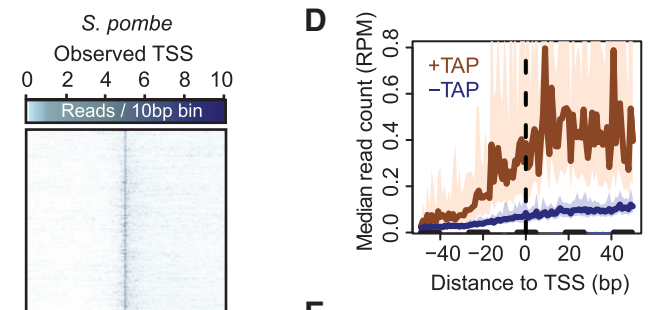

E

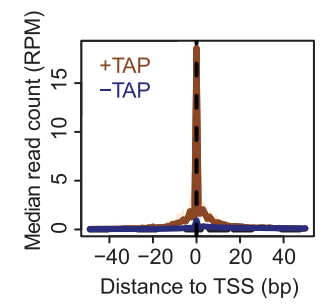

$\mathbf{F}$

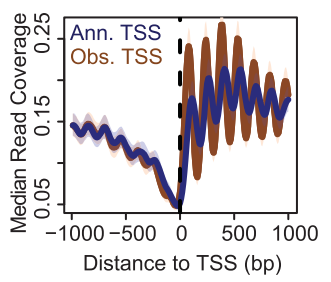

Figure 1. PRO-seq and PRO-cap capture transcription elongation and initiation genome-wide in S. pombe. ( $A$ ) Browser tracks of PRO-seq (plus strand: red; minus strand: blue) and PRO-cap data (plus show the reannotated, "observed" transcription start-sites based on PRO-cap data. Blue gene models correspond to the available annotations of genes. (B) Heatmaps of S. pombe PRO-cap signal for each base within $\pm 250 \mathrm{bp}$ around the annotated TSS (left) and PRO-cap observed TSS (right) for all active and filtered genes $(n=3214)$. Genes within heatmaps are sorted by increasing downstream distance notated TSS (left) or observed TSS (right) generated using WebLogo (Crooks et al. 2004). (D) PRO-cap signal from samples prepared either with or without Tobacco Acid Pyrophosphatase (TAP) treatment centered on annotated TSS. The TAP-minus samples represent empirical levels of background for each genomic position. $(E)$ Median PRO-cap signal from samples prepared either with or without TAP treatment centered on observed TSS. $(F)$ Median MNase-seq coverage centered on annotated TSSs (blue) or observed TSSs (brown). For the metagene plots, the $y$-axis shows the median read counts for each base-pair $(D, E)$, or median read coverage within 10-bp bins $(F)$. In $D, E$, and $F$, the $12.5 \%$ and $87.5 \%$ quantiles are shown in lightly shaded regions.

We refer to the selected base as the "observed TSS," and to previous annotations as the "annotated TSS." Heatmaps of PRO-cap read depth surrounding previously "annotated TSSs" in S. pombe show that initiation is distributed around the annotated TSS, but that the annotation rarely matches the base-pair resolution measurement of initiation that we make with PRO-cap (Fig. 1B, left panel). In contrast, the distribution of PRO-cap reads is centered and highly focused at our "observed TSSs" in S. pombe, indicating a narrow window within which start-site selection occurs (Fig. 1B, right panel). Moreover, a moderate sequence preference for 
initiating at an $A / G$, immediately downstream from a $C / T$, was revealed under the observed TSS, whereas no base preferences underlie the PomBase annotations for the same genes (Fig. 1C). More recently, groups have used other approaches to reannotate transcription units across the fission yeast genome (Li et al. 2015; Eser et al. 2016). Comparing our PRO-cap-defined annotations in S. pombe with TSSs determined by Eser et al. (2016) revealed a much closer agreement between these two independent approaches than with PomBase annotations, although some differences still remained (Supplemental Fig. S2A,B).

For S. cerevisiae, we compared our PRO-cap observed TSSs with the $5^{\prime}$ ends of the longest major transcript isoforms identified by Pelechano et al. (2013) (Supplemental Fig. S1). Although a striking enrichment of PRO-cap signal occurred directly over expected $5^{\prime}$ ends of these transcript isoforms (Supplemental Fig. S1B,D), we found many preferred TSSs to be more downstream, consistent with the existence of many shorter major transcript isoforms in $S$. cerevisiae (Supplemental Fig. S1B; Pelechano et al. 2013).

To assess the amount of signal attributable to background in our PRO-cap experiments, we simultaneously prepared PRO-cap libraries from the same starting material in which nascent RNAs were not treated with Tobacco Acid Pyrophosphatase (TAP) prior to $5^{\prime}$ adaptor ligations. These sequenced RNAs represent molecules lacking a $5^{\prime}$ cap structure and therefore are unlikely to represent true transcription start sites. Looking at a 100-bp window around annotated or observed TSSs in S. pombe, we observed a highly focused enrichment of reads over background centered on the observed TSS (Fig. 1E), a trend that does not exist when centering previous annotations (Fig. 1D). The same analysis in S. cerevisiae revealed PRO-cap signal highly consistent with the transcript isoform $5^{\prime}$ ends used, suggesting good agreement of our data with previous work (Supplemental Fig. S1D; Pelechano et al. 2013). Nonetheless, we attained an even greater reduction in signal dispersion by centering PRO-cap signal around the most preferred base (Supplemental Fig. S1E). Plotting PRO-seq signal relative to TSSs confirms that average 5 ' boundaries' global RNA polymerase profiles more closely reflect the observed TSS compared with previous annotations in both fission and budding yeast (Supplemental Fig. S3). Moreover, PRO-cap signal in S. cerevisiae was found to be strictly enriched downstream from previously characterized TATA-like motifs and TFIIB positions (Supplemental Fig. S4; Rhee and Pugh 2012).

To further evaluate our observed TSSs, we assessed the effect of centering available MNase-sequencing data around the preferred base (DeGennaro et al. 2013; Hu et al. 2014). Composite MNase-seq profiles around the observed TSSs in $S$. pombe show a more highly phased pattern when compared with annotated TSSs (Fig. 1F). Centering MNase-seq data from S. cerevisiae around the observed TSSs produced a modest reduction in phasing pattern of the MNase-seq reads, relative to the longest TIF-seq isoform 5 ' ends (Supplemental Fig. S1F). Together, our results indicate that PRO-cap-defined TSSs are truly an accurate representation of transcriptional start sites in $S$. pombe and $S$. cerevisiae that can improve our analysis of early transcription elongation in yeast, especially in $S$. pombe, where less mapping has been performed to date.

\section{Budding and fission yeast exhibit vastly different polymerase distributions across genes}

Using PRO-seq-based maps of RNA Pol II in Saccharomyces cerevisiae and Schizosaccharomyces pombe, we sought to identify general features of transcription that are species specific. To minimize the effects of transcription emanating from neighboring genes, we identified a set of filtered genes at least $1 \mathrm{~kb}$ from any other genes on the same strand (S. pombe: $n=874 ;$. cerevisiae: $n=$ 1101). We then generated scaled composite profiles in which the region between $300 \mathrm{bp}$ downstream from the observed TSS and $300 \mathrm{bp}$ upstream of the cleavage and polyadenylation signal (CPS) is scaled to the same number of bins, while everything outside this gene-center region is not scaled (10 bp bins). Importantly, PRO-seq signal increases immediately downstream from the observed TSS (Fig. 2A,B), further indicating PRO-cap-based observed TSSs are genuine start sites for these genes.

Similar to previous studies of transcription in S. cerevisiae (Steinmetz et al. 2006; Churchman and Weissman 2011), we observed a relatively uniform distribution of RNA Pol II across transcription units, with transcription extending $\sim 200-300$ bp beyond the CPS on average (Fig. 2A). Notably, NET-seq profiles exhibit a modest decline in read density downstream from the TSS, which was not present in our PRO-seq data (Supplemental Fig. S5A). This distinction may reflect differences in the forms of nascent RNA captured by each technique. For instance, NET-seq captures all Pol IIassociated RNAs with a $3^{\prime} \mathrm{OH}$, such as those in backtracked complexes. Pol II backtracking may occur more frequently during early elongation (Churchman and Weissman 2011) and not be captured by PRO-seq, which requires complexes capable of running on. Nonetheless, in contrast to the Pol II distributions of S. cerevisiae measured by either technique, our composite RNA Pol II profiles across genes in $S$. pombe show two more prominent peaks of Pol II density. The first peak is immediately downstream from the TSS, whereas a second, broader peak occurs downstream from the 3' CPS. Furthermore, Pol II in S. pombe appears to transcribe much farther beyond the CPS when compared with $S$. cerevisiae (Fig. 2B). Visualization of PRO-seq density for representative genes, YBR078W in S. cerevisiae and SPBC32F12.10 in S. pombe, illustrates these same characteristics (Fig. 2C,D). Regarding intron-exon junctions, Pol II density around fission yeast splice sites exhibited features similar to those described in Drosophila (Kwak et al. 2013), whereas the rarity of introns in budding yeast precluded a more thorough comparison (Supplemental Fig. S6A,B).

To compare the enrichment of RNA Pol II density downstream from the TSS between S. pombe and S. cerevisiae, we calculated a $5^{\prime}$ pausing index for each gene considered in the composite profiles. The $5^{\prime}$ pausing index was defined as the ratio of read density within the first $100 \mathrm{bp}$ downstream from the TSS over the gene body (TSS +200 to CPS). On average, S. pombe genes exhibited significantly higher pausing indexes when compared with $S$. cerevisiae (Fig. 2E). Similarly, to assess the amount of pausing or slowing beyond the $3^{\prime}$ CPS, we computed a 3 ' termination index for these filtered genes. The post-CPS termination index was defined as the ratio of the highest density $100 \mathrm{bp}$ window within $500 \mathrm{bp}$ downstream from the CPS to the gene body (defined above). We found that $S$. pombe genes on average also have a significantly higher termination index than $S$. cerevisiae.

To investigate the significance of promoter-proximal pauselike distributions observed in $S$. pombe, we systematically classified genes as being paused or not paused. Genes classified as paused were required to show a significant enrichment (Bonferroni corrected $P<0.01$ ) of mappable reads within the promoter-proximal region (TSS to $+100 \mathrm{bp}$ ) relative to the gene body, as assessed by Fisher's exact test. Not paused genes were required to have a $P$-value $>0.99$, suggestive of no enrichment of promoter-proximal reads. Based on the criteria above, of the 3214 filtered $S$. pombe genes (Methods), we identified 901 genes as being paused and 
A

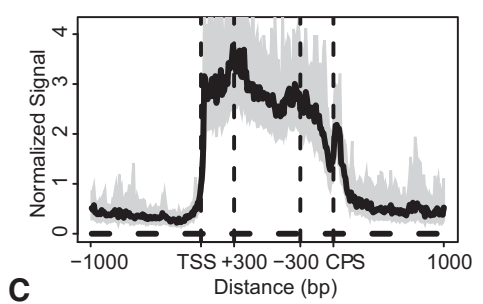

C

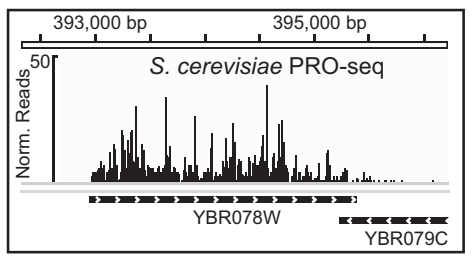

E

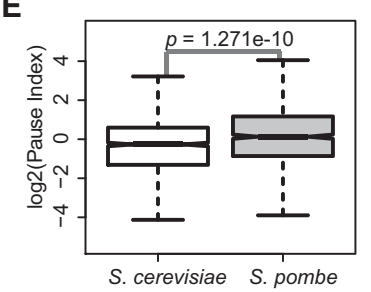

G

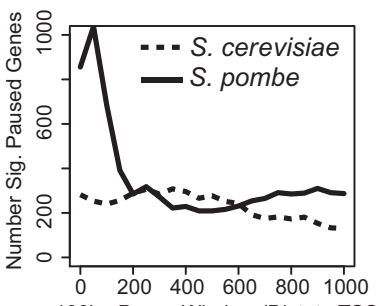

$100 \mathrm{bp}$ Pause Window (Dist. to TSS)

Figure 2. PRO-seq reveals distinct transcription elongation profiles in S. cerevisiae and S. pombe. $(A, B)$ Median PRO-seq read count across all active and filtered genes in S. cerevisiae $(A)$ or $S$. pombe $(B)$ that are separated from neighboring genes on the strand by at least $1 \mathrm{~kb}$ (S. cerevisiae: $n=1101$; S. pombe: $n=$ 874). Bins between the +300 and -300 bp marks are scaled based on gene length, whereas upstream of and downstream from this center region, 10-bp bins were used. The shaded regions around the curves represent the $12.5 \%$ and $87.5 \%$ quantiles. (C,D) Representative genes from S. cerevisiae $(C)$ and S. pombe $(D)$ with PRO-seq read counts plotted above. $(E, F)$ Box plots of pausing index $(E)$ or termination index $(F)$ values calculated for all genes that were included in $A$ and $B$. $P$-values represent results of Student's $t$-test. (G) A test for enrichment of pausing near the promoter versus other gene regions. Reads were counted within a sliding 100-bp window from 0 to 1000 bp from the TSS of all filtered, active genes and divided by the counts within the remaining mappable gene length. Fisher's exact test was used to determine the number of significantly paused genes (adjusted $P<0.01$ ). yeast (DeGennaro et al. 2013). Both data types showed broad Pol II enrichment downstream from the CPS for all gene types (Supplemental Fig. S5B, right). Furthermore, consistent with our gene classification based on PRO-seq, highly paused genes also showed a promoter-proximal Pol II ChIP-seq peak that was greater than that of the other gene groups, further suggesting an enrichment of elongating Pol II downstream from the TSS for these genes (Supplemental Fig. S5B, left).

To assess whether the observed pause peaks are specific to the promoter-proximal region, we repeated the calculation of pausing index and significance using a sliding 100-bp window starting every 50 bases between the TSS and $1000 \mathrm{bp}$ downstream (20 measurements per gene). We then tallied the number of genes that were identified as significantly paused within each window relative to the remaining gene length. In $S$. pombe, we found that the most promoter-proximal regions have significantly larger number of genes identified as paused, but in S. cerevisiae, there is not a significant difference in any given window (Fig. 2G). These results strongly indicate the existence of a previously undescribed form of promoter-proximal pausing in $S$. pombe that is absent in $S$. cerevisiae.

Average Pol II density across transcription units of fission and budding yeast reveals large, previously uncharacterized, differences in transcription mechanisms between these divergent yeast. Although our data in S. cerevisiae corroborate the wealth of available information describing Pol II distributions in this system (Churchman and Weissman 2011; Jordán-Pla et al. 2014), applying the same technique in $S$. pombe has identified Pol II dynamics that resemble mechanisms pervasive in metazoans.
2133 not paused. The relative amount of active genes identified as paused in $S$. pombe (28\%) is thus lower than that observed for humans (41\%), using the same criteria (Core et al. 2008). Although there were few functional class (Thomas et al. 2003) enrichments for paused or not paused genes, highly paused genes (defined below) were significantly enriched for structural constituents of the ribosome $\left(P=3.84 \times 10^{-4}\right)$, which are often highly paused in other species (Min et al. 2011).

Broad peaks of RNA Pol II have been observed beyond the 3' CPS of many genes in S. pombe (Coudreuse et al. 2010; Sansó et al. 2012; Castel et al. 2014); yet to our knowledge, no group has identified a prominent peak downstream from the TSS. We thus compared PRO-seq profiles for highly paused, not paused, and all genes with publicly available Pol II ChIP-seq data in fission
Paused and not paused genes in S. pombe exhibit distinct Pol II patterns around their +1 nucleosome

Having observed the distribution of RNA Pol II in fission yeast, we further investigated differences between paused and not paused genes. Paused and not paused genes, identified using the combined replicate data, were further refined as high-confidence paused and not paused genes by requiring that they are also significantly paused $(P<0.05)$ or not paused $(P>0.95)$ in each biological replicate (paused: $n=656$; not paused: $n=2088$ ). An example of PRO-seq profiles for a highly paused gene and a not paused gene are displayed in Figure 3, A and B, respectively. Average PRO-seq profiles centered on the observed TSS in S. pombe show a striking signal enrichment, specific to paused genes,

\section{Genome Research}

www.genome.org 
A

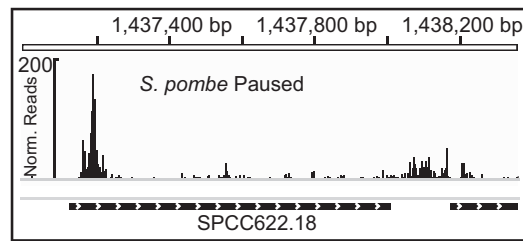

C

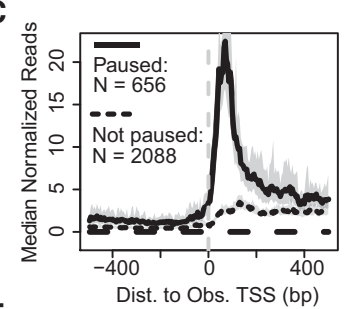

E

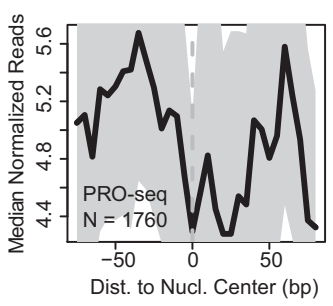

G

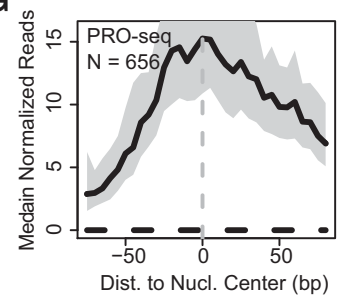

B

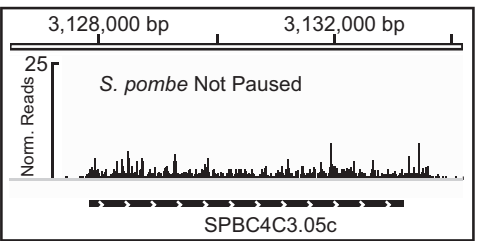

D

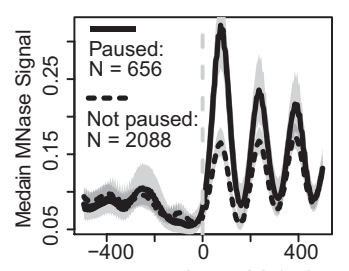

$\mathbf{F}$

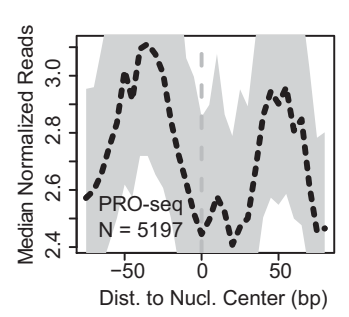

H

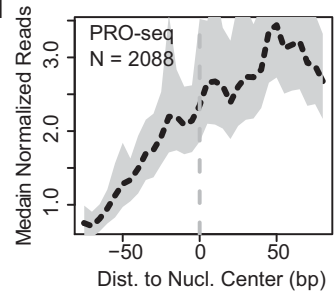

Figure 3. Pol II distributions at paused genes in S. pombe are coupled with increased nucleosome occupancy or positioning. $(A, B)$ Browser images of PRO-seq read counts across $S$. pombe genes classified as either high-confidence paused $(A)$ or not paused $(B)$. (C) Median PRO-seq read counts within $1 \mathrm{~kb}$ upstream of and downstream from the observed TSS of paused and not paused genes. (D) Median MNase-seq read coverage within $1 \mathrm{~kb}$ upstream of and downstream from the observed TSS of paused and not paused genes. $(E, F)$ PRO-seq signal around gene-body nucleosome centers for paused $(E)$ and not paused genes $(F)$. $(G, H)$ PRO-seq signal around +1 nucleosome centers for paused $(G)$ and not paused genes $(H)$. For metagene plots in $\mathrm{C}-\mathrm{H}$, medians reflect 5 -bp bins, and the $12.5 \%$ and $87.5 \%$ quantiles are shown as shaded regions. All panels represent profiles of combined wild-type biological replicates.

largely confined within the first $100 \mathrm{bp}$ downstream from the TSS (Fig. 3C).

The transcription process is tightly coupled to the chromatin environment (Venkatesh and Workman 2015), and nucleosomes are known to form a barrier to transcriptional elongation, slowing or stalling RNA polymerases as they unwind the coiled DNA around the histone octamer (Hall et al. 2009). We analyzed an existing MNase-seq data set to explore the organization of nucleosomes relative to Pol II initiation and pause sites. Interestingly, average MNase profiles relative to the observed TSSs revealed a more highly phased nucleosome pattern for paused genes than for not paused genes (Fig. 3D). The largest difference in MNaseseq signal was at the +1 nucleosome position, possibly suggesting that this nucleosome is more precisely positioned or maintains higher occupancy within paused genes.

To assess the distribution of elongating polymerases around nucleosomes in S. pombe, we displayed average PRO-seq density relative to previously identified nucleosome centers (Givens et al. 2012). For both paused and not paused genes, we parsed nucleosomes within gene boundaries based on their relative positions to the TSS. Nucleosomes were split into two groups: +1 nucleosomes (first nucleosome downstream from TSS) and genebody nucleosomes (nucleosomes downstream from +1 center). Within the gene body, Pol II shows a similar profile around nucleosomes whether the gene is paused or not paused (Fig. 3E,F). The distributions around gene-body nucleosomes reflect known properties of transcription through chromatin, where Pol II slows down when it encounters the strong force of the DNA histone interaction, and speeds up when those contacts are broken as it approaches the DNA near the dyad (Hall et al. 2009). Plotting the average PRO-seq density within $160 \mathrm{bp}$ around +1 nucleosome centers for paused and not paused genes revealed a remarkable difference in Pol II distribution relative to the dyad axis. Whereas Pol II shows no enrichment upstream of the +1 nucleosome center within not paused genes (Fig. 3H), Pol II is highly enriched around the dyad axis of the +1 nucleosome of paused genes (Fig. 3G). This data may suggest a role for histoneDNA interactions in specifying pause sites in S. pombe. Conversely, Pol II pausing may be independent of the nucleosome barrier, but restrict movement of the +1 nucleosome (Struhl and Segal 2013), inducing a more rigid positioning of downstream nucleosomes also (Jiang and Pugh 2009). The Pol II distributions around the +1 and gene-body nucleosomes $(+2$ to +4$)$ indicate a general difference between the way Pol II negotiates its transit through the first and subsequent nucleosomes, as well as an interplay between paused Pol II and the +1 nucleosome that distinguishes paused and not paused genes.

\section{Deletion of Spt4 increases Pol II density genome-wide in both budding and fission yeast}

The striking resemblance of Pol II distributions in S. pombe to promoter-proximal pausing in metazoans led us to postulate that there is a pausing factor conserved in yeast playing a role in this process. Because both $S$. cerevisiae and $S$. pombe lack NELF subunits, the next logical candidate was the Spt4-Spt5 complex. To investigate functional divergence of this complex between $S$. cerevisiae and $S$. pombe, we prepared PRO-seq libraries from strains lacking Spt4. Wild-type and spt4 $\Delta$ PRO-seq libraries from each species were prepared from two biological replicates. The absence of all 
transcription and messenger RNA from the Spt4 locus confirms the deletion of the gene in each yeast (Supplemental Fig. S7A,B).

Anticipating the possibility of global transcriptional changes upon deletion of Spt4, we used an approach to normalize sequencing depth between samples based on a spike-in reference control. In this approach, we took advantage of the fact that $>99 \%$ of mappable $36-n t$ sequences derived from $S$. pombe and $S$. cerevisiae can be distinguished. Similar to an approach developed previously for microarray analysis of labeled mRNA (Sun et al. 2012), we included an exogenous, whole yeast spike-in control in our experiments, which was carried out as follows: All samples and biological replicates to be compared were required to have precisely the same starting $\mathrm{OD}_{600}$ before harvesting. Immediately prior to harvesting, a fixed volume of the divergent "spike-in" yeast culture was added to each sample. After preparing and sequencing PRO-seq libraries from the mixed samples, we aligned sequencing reads to a combined genome of $S$. cerevisiae and $S$. pombe with the requirement that sequences align to only one location to remove any ambiguous reads. The proportion of spike-in to experimental reads was highly similar between biological replicates (Supplemental Fig. S7G,H), and normalization factors were calculated from the relative number of uniquely mapped reads from the spiked-in genome between samples (Supplemental Table S1). To verify that this approach accurately scales the sequencing depth of each sample, we compared the normalized gene-body read densities between biological replicates, resulting in Spearman's $\rho>0.97$ (Supplemental Fig. S7C-F). Importantly, replicate comparisons are nearly symmetrical about the diagonal line, $x=y$, indicating this normalization accurately accounts for any technical differences in library preparation and sequencing.

To assess the effects of deletion of Spt 4 on transcription in $S$. cerevisiae and $S$. pombe, we performed a differential expression analysis using read counts within the gene-body region. After spike-in normalization, we observed a global increase in the gene-body read density in spt4 $\Delta$ strains in both yeasts. Using the DESeq2 R package (Love et al. 2014), we identified 1572 genes in S. cerevisiae (46\% of filtered, active genes) and 2291 genes in $S$. pombe (67\%) as having significantly higher gene-body Pol II levels $(P<0.01)$ (Fig. 4A,B). In contrast, only $47(1.4 \%)$ and $156(4.8 \%)$ genes were found to have significantly lower read counts in $S$. cerevisiae and $S$. pombe, respectively. On average, Pol II density within gene bodies of $S$. pombe and $S$. cerevisiae transcription units increased by a factor of 1.81 and 1.47 , respectively. Looking more specifically at the YGR204W locus in S. cerevisiae (Fig. 4C) and the SPCC622.18-1 locus in S. pombe (Fig. 4D), we clearly observed a reproducible increase in the signal intensity within the gene body in $s p t 4 \Delta$ biological replicates compared with wild type. Thus, the use of spike-in controls in the preparation of these data is invaluable. This approach circumvents the assumptions of an equal number of RNA sequence reads per cell or that genes on average are unchanged, both of which are violated in mutants globally affecting transcription (Sun et al. 2012).

Our spike-in-normalized PRO-seq data also lends itself to a precise analysis of noncoding RNA. Antisense transcription within the gene body, as well as upstream, divergent transcription at promoters has become increasingly appreciated in yeast with the advent of high-throughput assays of RNA (Wilhelm et al. 2008; Neil et al. 2009; Xu et al. 2009). Evidence for alterations in such noncoding transcription have been reported for mutations in the elongation factor, Spt6, in S. pombe (DeGennaro et al. 2013). Subsets of genes used in the analysis of upstream, antisense or intragenic, antisense transcription were filtered to remove possible influence of nearby functional transcription. Unlike what has been observed in mammals (Core et al. 2008), composite profiles of PRO-cap and PRO-seq density around transcription start sites showed little indication of widespread upstream, divergent transcription at promoters in either yeast (Supplemental Fig. S8A,B,E, F), although $S$. cerevisiae tended to have higher ratios of upstream, antisense to downstream, sense transcription than $S$. pombe (cf. Supplemental Fig. S8C,G). Interestingly, intragenic, antisense to sense transcription ratios were more affected in $S$. pombe than in S. cerevisiae by the deletion of Spt4 (cf. Supplemental Fig. S8D,H). Indeed, we found that most levels of antisense transcription in fission yeast were unchanged (71\%) or decreased (19\%) in spt4 4 , suggesting that in $S$. pombe, antisense Pol II transcription may not depend on Spt4 in the same manner.

The general increase in sense-transcribing Pol II levels within a large proportion of genes likely reflects the ubiquitous role of Spt4 in positively affecting transcription processivity and elongation rate in these divergent yeasts (Wada et al. 1998; Rondón

\section{Genome Research}

www.genome.org 
et al. 2003; Hartzog and Fu 2013). Elevated levels of RNA abundance are often interpreted as a higher level of gene activity or increased initiation rates. However, increases in Pol II density could also reflect a reduction of Pol II elongation rate in the case of a fixed initiation rate (Danko et al. 2013; Jonkers et al. 2014).

\section{Genome-wide increases in nascent transcription in spt4 $\Delta$ are not reflected in mRNA abundance}

To test the possibility of a global elongation rate defect in $S$. cerevisiae and $S$. pombe lacking Spt4, we investigated the effects of the mutation on mRNA abundance. We postulated that under conditions in which Pol II experiences a compromised elongation rate, with little change in recruitment and initiation, messenger RNA would continue to be made at roughly the same rate. To assay the relative abundance of all expressed transcripts, we prepared strand-specific poly-A selected RNA-seq libraries from the same wild-type and $s p t 4 \Delta$ strains of budding and fission yeast. Again, to control for the possibility of genome-wide differences in transcript abundance per cell, we used the same culture-level divergent yeast spike-in approach as used for the PRO-seq experiments (Supplemental Table S1). The deletion of Spt4 was confirmed by absence of any reads from the locus (Supplemental Fig. S7A,B), and mRNA levels were found to be highly reproducible between biological replicates (Supplemental Fig. S9A-D).

Using the gene-body region to quantify gene activity from PRO-seq or mRNA-seq data, we observed a moderate correlation between nascent transcription and mRNA abundance in wild-type strains (S. cerevisiae: Spearman's $\rho=0.572 ; S$. pombe: Spearman's $\rho=0.574$ ) (Supplemental Fig. S9E,F). We next asked if changes in transcription, resulting from the deletion of Spt4, consequently affect mRNA abundance. Here, we compared the distribution of fold changes in gene-body read-density from spt4 $\Delta$ to wild-type libraries between PRO-seq and mRNA-seq experiments. Although PRO-seq signal increases within gene bodies upon deletion of Spt4 in both species, changes in mRNA levels were either largely unchanged (S. cerevisiae) or slightly reduced (S. pombe) (Fig. 5A,B). Although the deletion of Spt4 altered mRNA abundance of some genes in both species (Supplemental Fig. S10A,B), these changes in gene-body read density were poorly correlated with changes in PRO-seq density (S. cerevisiae: Spearman's $\rho=$ 0.248; S. pombe: Spearman's $\rho=0.359$ ) (Supplemental Fig. S9G, $\mathrm{H})$. Overall, the effects of deleting Spt4 on Pol II density within a gene is in stark contrast with the messenger RNA abundance, sup-
A

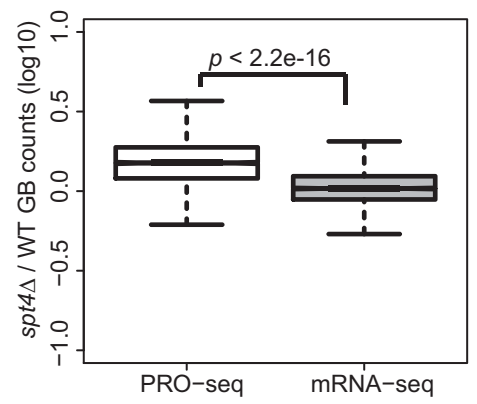

B

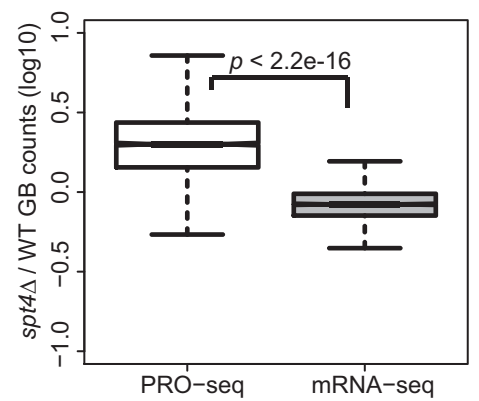

Figure 5. Global increase in Pol II density in spt4 $\Delta$ does not result in increased transcript abundance in S. cerevisiae or S. pombe. $(A, B)$ Box plots comparing the fold change in gene-body PRO-seq density resulting from the deletion of Spt4 with the corresponding change in poly-A selected RNA-seq in S. cerevisiae $(A)$ and S. pombe (B). P-values represent the results of Student's $t$-test. porting a model in which Spt4 increases the elongation rate of RNA Pol II transcription in vivo without necessarily altering Pol II recruitment or initiation.

To determine whether the effects of Spt 4 deletion in S. pombe depended on the extent of observed promoter-proximal pausing, we binned genes in deciles based on the magnitude of their pausing index. Gene characteristics, such as expression level and termination index, appeared to be unrelated to pausing index (Supplemental Fig. S11A,B,E). Furthermore, there were no considerable relationships between how paused a gene is and the effect of Spt4 deletion on gene-body Pol II density (Supplemental Fig. S11C) or mRNA abundance (Supplemental Fig. S11D). We surmise that Spt4, as a complex with Spt5, influences various transcriptional features independent of the degree of promoter-proximal pausing.

\section{Deletion of Spt4 reduces promoter-proximal pausing and elongation beyond the cleavage and polyadenylation signal in S. pombe}

Although in metazoans, the Spt4-Spt5 complex acts as a pausing factor as well as a positive elongation factor (Wada et al. 1998; Wu et al. 2003), most research in yeast has focused on its role in positively influencing transcription (Hartzog and Fu 2013). To assess the existence of additional roles for the Spt4-Spt5 complex in yeast, we investigated the effect of Spt 4 deletion on the distribution of Pol II at gene $5^{\prime}$ and $3^{\prime}$ ends in both species. To limit the effects of nearby genes, we restricted all analyses in this section to genes $>1 \mathrm{~kb}$ in length and at least $1 \mathrm{~kb}$ away from neighboring genes on the same strand (S. pombe: $n=714 ; S$. cerevisiae: $n=$ 816). Plotting the median PRO-seq signal around the observed TSS of $S$. pombe genes for wild-type and $s p t 4 \Delta$ strains revealed a dramatic shift of the Pol II density downstream in mutants (Fig. 6A). Whereas wild-type PRO-seq profiles display the pause-like distributions immediately adjacent to the TSS, $s p t 4 \Delta$ strains lack this focused, promoter-proximal peak. Instead, they display a more gradual accumulation of density that is much greater than that of the wild type within the gene body. To evaluate the generality of this phenotype for all 714 genes, we generated heatmaps displaying the fold change in PRO-seq read density $\left(\log _{2}[s p t 4 \Delta /\right.$ WT]) within $10 \mathrm{bp}$ bins from $-1 \mathrm{~kb}$ to $+1 \mathrm{~kb}$ around the observed TSS (Fig. 6B). We found that the majority of genes in the mutant show a decreased level of Pol II relative to wild type solely within the first $\sim 150$ bases. Beyond this promoter-proximal region, spt4A strains showed heightened PROseq density compared with wild type. Furthermore, this mutant enrichment is most pronounced early within the gene body, as if early elongation complexes were leaking into the downstream regions rather than undergoing a pause. To quantify the significance of this shift in Pol II density, we calculated a pausing index for each gene in wild-type and $s p t 4 \Delta$ strains. Consistent with the changes in average PRO-seq profiles in Figure $6 \mathrm{~A}$, we observed a highly significant decrease in average pausing index for all genes used in this analysis (Fig. 6C).

In light of the fact that Spt4-Spt5 tracks with elongating Pol II across the entire length of genes in eukaryotes 
A
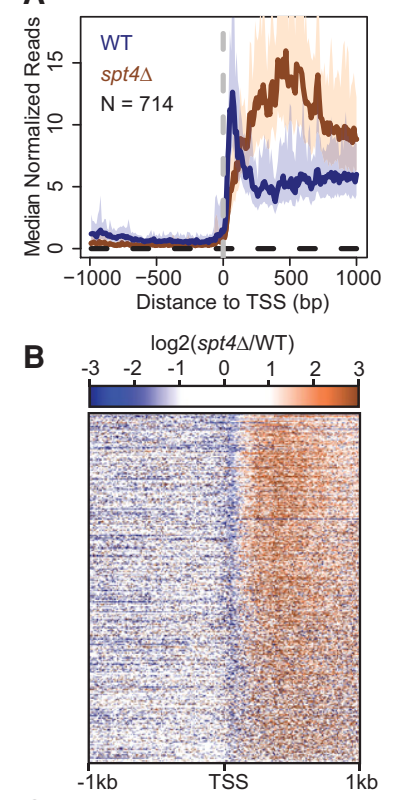

C

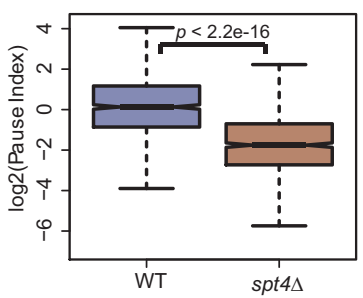

D

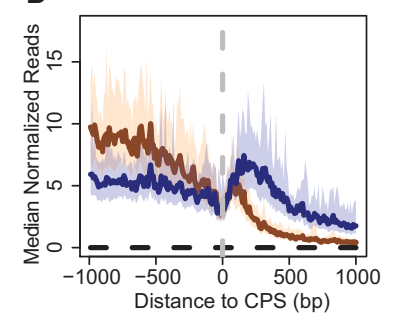

E $\quad-3 \quad-2^{\log 2(s p t 4 \Delta / \mathrm{WT})}$

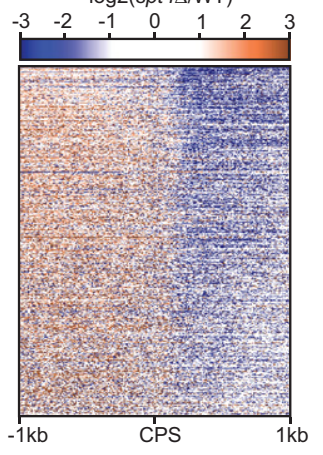

$\mathbf{F}$

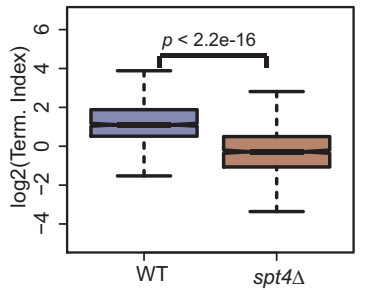

Figure 6. $5^{\prime}$ and $3^{\prime}$ ends of genes exhibit a loss of Pol II density as a result of Spt4 deletion in S. pombe. $(A, D)$ Median PRO-seq signal around the observed TSS $(A)$ or CPS $(D)$ of active, filtered genes longer than $1 \mathrm{~kb}$ and separated from the boundaries of neighboring genes on the same strand by at least $1 \mathrm{~kb}$. Medians reflect 10 -bp bins, and the $12.5 \%$ and $87.5 \%$ quantiles are shown in light shaded regions. $(B, E)$ Heatmaps of $\log _{2}$ fold change of mutant versus wild type per 10-bp bin around the TSS $(B)$ or CPS $(E)$ for all genes used in $A$ and $D$. Genes within heatmaps are sorted by decreasing amount of wild-type PRO-seq reads within the first 500 bp downstream from the TSS. (C) Box plots showing the distribution of pausing index values for WT and spt4 $\Delta$ in S. pombe. (F) Box plots showing the distribution of termination index values for WT and spt $4 \Delta$ in $S$. pombe. $P$-values represent the results of Student's $t$-test.

(Mayer et al. 2010; Rahl et al. 2010), we were curious about the effect of Spt4 deletion on transcription around the cleavage and polyadenylation signal (CPS). Similar to our analysis of PRO-seq signal around the observed TSS, we calculated the median read density around the annotated CPS for all 714 genes. Strikingly, although the spt4s S. pombe strain shows elevated levels of elongating Pol II leading up to the CPS, immediately after this element, we begin to observe a much more rapid decline in polymerase density in the mutants relative to wild type (Fig. 6D). Looking at fold change in polymerase density across this surrounding region for each individual gene shows that this decreased transcription beyond the CPS in $s p t 4 \Delta$ is broadly observable (Fig. $6 \mathrm{E}$ ). Finally, we assessed the significance of this change in post-CPS transcription by calculating a termination index for each gene in wild-type and spt4A $S$. pombe strains. The combined effect of higher read density within the gene body and lower signal beyond the CPS in spt4A, produced a significantly lower average termination index relative to wild

type (Fig. 6F). Thus, in fission yeast, transcription beyond the CPS is dramatically reduced in the absence of Spt4.

Profiles of transcription elongation differ greatly between budding and fission yeast, yet upon deletion of Spt4, both show a similar increase in Pol II levels within gene bodies. To address whether $S$. cerevisiae is similarly affected by deletion of Spt 4 at the $5^{\prime}$ and $3^{\prime}$ gene boundaries, we performed the same set of analyses on our PRO-seq samples from wild-type and spt4 $\Delta$ strains (Supplemental Fig. S12). Surprisingly, genes in S. cerevisiae exhibit an enormous increase in PRO-seq density at the $5^{\prime}$ ends of genes upon deletion of Spt4 (Supplemental Fig. S12A,B). This is precisely the opposite effect of that observed in S. pombe and results in a significant increase in average pausing index (Supplemental Fig. $\mathrm{S} 12 \mathrm{C}$ ). Beyond the CPS, somewhat similar to $S$. pombe, there appears to be a slight reduction in PRO-seq signal as a result of Spt4 deletion (Supplemental Fig. S12D,E). However, this modest reduction does not register as a significant reduction in average termination index (Supplemental Fig. S12F).

The highly contrasting effect of Spt 4 deletion in budding and fission yeast at the $5^{\prime}$ ends of genes indicates divergent roles for this elongation factor in early transcription elongation. Whereas Spt4 appears to be solely required to enhance productive elongation in S. cerevisiae, our results in $S$. pombe reveal an additional function of Spt4 in promoting early Pol II pausing. Perhaps as evidence of shared defects in elongation, Pol II requires Spt 4 for extended transcription beyond the CPS in both species.

\section{Discussion}

We have adapted the Precision Run-On sequencing (PRO-seq) and $5^{\prime}$ cap sequencing (PRO-cap) protocols for use in both budding and fission yeast. Precisely mapping 5' ends of capped nascent RNAs reveals highly preferred transcription start sites for a large fraction of coding genes and noncoding transcription units, enhancing current annotations for many applications. Our PRO-seq profiles provide comprehensive base-pair resolution maps of elongating RNA polymerases genome-wide in two evolutionarily distant yeast strains. These data provide the highest resolution, strand-specific look at transcription across the fission yeast genome to date.

The detailed transcriptional landscapes of $S$. cerevisiae and $S$. pombe prove to be very dissimilar, with metazoan-like features of transcription unique to fission yeast. Consistent with previous investigations of transcription in S. cerevisiae, Pol II across annotated genes is largely confined within the well-annotated $5^{\prime}$ and $3^{\prime}$ gene boundaries, with a relatively uniform distribution across transcription units (Steinmetz et al. 2006). In contrast, we found S. pombe to exhibit elongation continuing well beyond the $3^{\prime}$ cleavage and polyadenylation signal of most genes. Immediately downstream from the $S$. pombe $3^{\prime}$ CPS, Pol II appears to experience an elongation rate-reducing step, resulting in heightened polymerase density over a broad region. Although delayed termination is less well characterized in yeast, studies in a wide variety of metazoans have revealed similar patterns (Core et al. 2008, 2012; Kruesi et al. 2013; Jonkers et al. 2014). Our findings support the conclusion that the kinetics of transcription termination and its regulation in $S$. pombe is more similar to that of metazoans than S. cerevisiae.

Another intriguing characteristic of transcription profiles in fission yeast is the prominent enrichment in Pol II immediately downstream from the TSS, resembling promoter-proximal pausing in metazoans (Fuda et al. 2009; Adelman and Lis 2012). We found that $28 \%$ of our filtered, actively transcribed $S$. pombe genes could

\section{Genome Research}

www.genome.org 
be classified as significantly paused when considering the first 100 transcribed bases. These paused genes tend to show a much more highly positioned or high-occupancy +1 nucleosome, with Pol II density peaking near the dyad axis. Interestingly, this near-overlapping association of Pol II with the +1 nucleosome dyad is unlike that observed in Drosophila, in which most pausing occurs upstream of the +1 nucleosome (Kwak et al. 2013). This difference could be the result of NELF, which might have evolved in metazoans to capture Pol II rapidly in a more promoter-proximal paused state (Li et al. 2013) and thereby strengthen the potential for upstream factor regulation. Considering the proximity of the $+1 \mathrm{nu}-$ cleosome to the TSS and the tight relationship between the Pol II and nucleosome occupancy within this region in $S$. pombe, it is tempting to speculate that they are connected (Fig. 3G). On one hand, the first nucleosome may act as a barrier to early transcription elongation, causing a slower rate of transcription shortly after initiation (Weber et al. 2014). Alternatively, a frequently paused polymerase at this position could restrict the localization of the +1 nucleosome (Struhl and Segal 2013). A rigidly positioned $+1 \mathrm{nu}-$ cleosome could then restrict the mobility of downstream, genebody nucleosomes, resulting in the observed increase in phasing (Fig. 3D; Jiang and Pugh 2009).

In metazoans, two complexes, NELF and DSIF (Spt4-Spt5), are both critical for pausing (Wu et al. 2003). The observation of pause-like distributions in fission yeast is thus curious because $S$. pombe lack any recognizable NELF subunits that might help to establish a pause (Narita et al. 2003). Both S. pombe and S. cerevisiae, however, possess Spt4-Spt5 and CDK9 (Bur1) homologs (Hartzog and $\mathrm{Fu}$ 2013). Intriguingly, deleting Spt 4 in $S$. pombe causes a dramatic reduction in Pol II density within the promoter-proximal region. Moreover, heightened Pol II density shifts into the gene body, suggesting Spt4, most likely in complex with Spt5, is necessary for preventing the premature release of the Pol II from these sites during early transcription elongation. This possibility is supported by findings in metazoans indicating pausing is only modestly reduced upon RNAi knock down of NELF (Core et al. 2012; Gilchrist et al. 2012).

The apparent similarities between $S$. pombe and metazoan pausing raise many additional mechanistic and functional questions. As in metazoans, Spt4-Spt5-mediated promoter-proximal pausing in $S$. pombe might provide a regulatory step or checkpoint to ensure proper capping of the nascent RNA or modification of the elongation complex (Pei and Shuman 2002; Schwer et al. 2009; Viladevall et al. 2009; Schneider et al. 2010). Indeed, CDK9 and its Cyclin partner Pch1 in fission yeast interact with nascent RNA 5' capping machinery (Pei et al. 2003, 2006). Alternatively, paused Pol II could be capable of holding certain $S$. pombe genes poised for expression, synchronizing the expression of sets of genes, or allowing the integration of regulatory signals at distinct steps of recruitment and release of paused Pol II (Adelman and Lis 2012). In the future, determining whether pause release in $S$. pombe relies on the kinase activity of CDK9 will be imperative for comparing this phenomenon with that of metazoans.

Because Spt4 is a subunit of a ubiquitous elongation factor and can affect transcription broadly, we found that proper normalization of our PRO-seq and mRNA-seq data was critical for accurately assessing genome-wide changes in transcription between wild-type and mutant yeast strains. Using a cell culture spike-in approach, we observed global increases in sense-transcribing RNA polymerase density across genes in $\operatorname{spt} 4 \Delta$ strains of $S$. cerevisiae and $S$. pombe relative to wild type. However, effects on antisense transcription appeared to be species specific. In S. cerevisiae, anti- sense-to-sense ratios were minimally affected by the deletion of Spt4. In contrast, the relative amount of antisense transcription in $S$. pombe appeared to globally decrease, perhaps indicating little influence of Spt4-Spt5 over cryptic, intragenic, antisense transcription events in fission yeast. We found that mRNA levels in budding and fission were largely either unchanged or slightly decreased in the absence of Spt4, despite the increase in gene-body Pol II (Supplemental Fig S9). One explanation could be that RNAs may in fact be increasingly produced in spt4 $\Delta$ strains, yet buffered through surveillance mechanisms such as preRNA degradation. However, in light of several lines of evidence, we favor a hypothesis in which, in the absence of Spt4, Pol II exhibits a reduced elongation rate. First, early experiments demonstrated that recombinant human Spt4 and Spt5, but not Spt5 alone, could increase rates of in vitro transcription reactions (Wada et al. 1998). Second, reduced elongation rates are known to decrease the distance transcribed by Pol II beyond the 3 ' cleavage and polyadenylation signal (CPS) (Fong et al. 2015). We found that in S. pombe, transcription termination occurs within a much shorter distance from the CPS in the absence of Spt4 (this was also observed to a lesser extent in $S$. cerevisiae, but may be more difficult to detect because termination occurs normally only a short distance from the CPS). Compatible with the torpedo model of transcription termination (Connelly and Manley 1988), the $5^{\prime}-3^{\prime}$ exonucleases, Dhp1 (S. cerevisae: Rat1), may be better able to catch up to and displace the slower Pol II from the DNA. Importantly, since Spt 4 acts in complex with Spt5 to affect elongation, we surmise that many of the phenotypes we have observed in $s p t 4 \Delta$ strains are a consequence of perturbation of this elongation factor complex and its association with Pol II, rather than the independent action of Spt4. Further experiments are required to distinguish independent roles of each subunit of the Spt4-spt5 complex in transcription elongation.

Apart from having a role in maintaining transcription elongation rates, the Spt4-Spt5 complex is also thought to affect the processivity of Pol II (Mason and Struhl 2005; Hartzog and Fu 2013). The increase in gene-body Pol II in $s p t 4 \Delta$ strains does not preclude the possibility of defects in Pol II processivity, but rather indicates that the more dominant effect of Spt 4 deletion is on Pol II elongation rate. The modest decreases in levels of many mRNAs in $S$. pombe could reflect a combination of decreased rates of transcription and reduced processivity by Pol II in the absence of Spt4.

Using PRO-seq, adapted for determining the transcription profiles in budding and fission yeast, provides novel insight into the evolution of mechanisms governing their transcriptional landscapes. In this study, we identified divergence in the role of Spt4Spt5 between budding and fission yeast. S. cerevisiae appears to rely on Spt4 primarily to maintain a steady and rapid elongation rate. In contrast, $S$. pombe additionally possesses a previously undescribed role for this complex in slowing or stalling Pol II between the TSS and the +1 nucleosome. The ability with which PRO-seq can be applied in yeast ultimately enhances our ability to combine genome-wide snapshots of transcription elongation with the powerful genetic tools inherent to these model systems.

\section{Methods}

\section{Yeast strains}

S. cerevisiae strains, WT and $\operatorname{spt} 4 \Delta$, are in the background W303-1a and W303-1 $\alpha$, respectively. WT and spt4 $\Delta$ strains in $S$. pombe are in the background $972 \mathrm{~h}$-. 


\section{Precision Run-On sequencing in yeast}

The protocol described in Kwak et al. (2013) was modified slightly to accommodate the physiology of yeast (García-Martínez et al. 2004). Instead of nuclei isolation, both $S$. cerevisiae and S. pombe were permeabilized to allow for the efflux of endogenous NTPs and uptake of biotin-11-NTPs (Epicentre). Yeast cultures, grown overnight, were diluted to an $\mathrm{OD}_{600}=0.1$ and grown to mid-log phase $\left(\mathrm{OD}_{600}=0.5-0.6\right)$ in either YPD (S. cerevisiae) or YES media (S. pombe). Ten-milliliter cultures of equal cell concentration were spun down and media was removed. Cells were then resuspended in $10 \mathrm{~mL}$ cold $\mathrm{H}_{2} \mathrm{O}$. Cultures were pelleted again, resuspended in $10 \mathrm{~mL} 0.5 \%$ sarkosyl at $4^{\circ} \mathrm{C}$, and incubated on ice for $20 \mathrm{~min}$. Cells were then spun at a reduced RCF $(400 \mathrm{~g})$ for $5 \mathrm{~min}$ at $4^{\circ} \mathrm{C}$. After removal of permeablization buffer, yeast pellets were resuspended in $120 \mu \mathrm{L}$ of $2.5 \times$ transcription buffer $(50 \mathrm{mM}$ Tris $-\mathrm{HCl}, \mathrm{pH} 7.7,500 \mathrm{mM}$ KCL, $12.5 \mathrm{mM} \mathrm{MgCl}_{2}$ ) with $6 \mu \mathrm{L} 0.1$ M DTT and $3.75 \mu \mathrm{L}$ of each $1 \mathrm{mM}$ biotin-NTP added immediately before use. After suspending yeast in the aforementioned transcription mix, the volume was brought to $285 \mu \mathrm{L}$ with DEPC-treat-

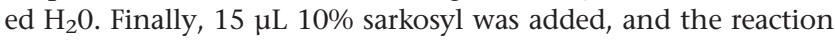
was placed at $30^{\circ} \mathrm{C}$ and allowed to run on for $5 \mathrm{~min}$. RNA was extracted using a hot phenol approach (Collart and Oliviero 2001); after the run-on reaction, cells were pelleted at $400 \mathrm{~g}$ for $5 \mathrm{~min}$ at $4^{\circ} \mathrm{C}$ and quickly resuspended in $500 \mu \mathrm{L}$ acid phenol. An equal volume of AES buffer (50 mM NaAc pH 5.3, $10 \mathrm{mM}$ EDTA, 1\% SDS) was added and placed for $5 \mathrm{~min}$ at $65^{\circ} \mathrm{C}$ with periodic vortexing, followed by $5 \mathrm{~min}$ on ice. Two hundred microliters chloroform was added and mixed followed by centrifugation at $14,000 \mathrm{~g}$ for $5 \mathrm{~min}\left(4^{\circ} \mathrm{C}\right)$. To the aqueous layer $(200 \mathrm{mM}), 3 \mathrm{M} \mathrm{NaOAc}$ was added followed by ethanol precipitation with a $3 \times$ volume of $100 \%$ ethanol. The RNA pellet was air dried before being resuspended in $20 \mu \mathrm{L}$ DEPC-treated water. The PRO-seq or PRO-cap protocol (Kwak et al. 2013) was then followed, beginning with base-hydrolysis, through to sequencing.

\section{Spike-in approach for Run-On (PRO-seq) or RNA isolation (RNA-seq)}

Preparation of libraries with a spiked-in reference organism was carried out by first growing a culture of the reference organism separate from the experimental cultures to $\mathrm{OD}_{600}=0.5$. After experimental samples had reached the desired $\mathrm{OD}_{600}$ and all samples were adjusted to have precisely equal numbers of cells, a fixed amount of the reference culture was added to each sample for a given experiment. All samples and biological replicates for a given experiment were prepared side by side such that all replicates received exactly the same amount of reference culture. In the case of either RNA-seq or PRO-seq, the spike-in approach described above was applied in order to control for every step of the sequencing library preparation during which technical variation may be introduced, beginning with the pelleting of cell cultures.

\section{PRO-seq/ cap sequencing alignment}

Raw sequencing files were processed by first removing reads that do not pass Illumina's quality filter. Full-length or partial adapter sequences (5'-TGGAATTCTCGGGTGCCAAGG-3') were then removed using FASTX-Toolkit (http://hannonlab.cshl.edu/ fastx_toolkit/). Reads were then trimmed to a maximum length of $36 \mathrm{nt}$ (minimum: $15 \mathrm{nt}$ ) and, in the case of PRO-seq, reversecomplemented using the FASTX-Toolkit. Finally, all nascent sequencing alignments were performed using Bowtie (version 1.0.0) (Langmead et al. 2009). Processed reads were aligned allowing for two mismatches, while requiring a unique alignment to the genome. For PRO-seq experiments, due to the existence of reads originating from separate species resulting from our spike-in procedure, reads were initially aligned to ribosomal DNA sequences from each species. Because of the high level of conservation of these sequences, ribosomal mapping reads were not considered in a species-specific manner. The remaining reads were then mapped to a combined genome consisting of all chromosomes from both $S$. cerevisiae (sacCer3=S288C_reference_genome_R641-1_20110203) and $S$. pombe (version: ASM294v2) facilitating the removal of reads with an ambiguous origin. Although we are aligning reads of various lengths (15-36 nt), the majority of reads in each library were $36 \mathrm{nt}$ long. The method of aligning to a combined $S$. pombe and $S$. cerevisiae genome prevents genome-indistinguishable, shorter reads from being included in downstream analysis. Unique reads were parsed based on their species of origin. Bedgraph files were created by recording only the most 3 ' base of each read, which represents the position of the Pol II active site, for PRO-seq data, or the most $5^{\prime}$ base of reads from PRO-cap data. The counts at each position in the bedgraph file were normalized based on the relative amount of reads aligning to the spike-in genome (PRO-seq), or based on the number of reads per million mapped reads (PRO-cap) (for displaying in genome browsers). Bedgraphs were ultimately converted to bigWig-formatted files for downstream analysis.

\section{Preparation and alignment of RNA-seq libraries}

Total RNA was isolated from each sample culture (with spike-in reference) using the hot phenol approach followed by ethanol precipitation. The integrity of each sample was assessed on a denaturing poly-acrylamide gel ( $7 \mathrm{M}$ urea PAGE), and the quantity of total RNA was measured. Three micrograms of total RNA from each sample was used for library preparation. Sequencing libraries were prepared using the TruSeq Stranded mRNA LT Sample Prep Kit (Illumina). Raw sequencing of $75 \mathrm{nt}$ reads was conducted, and raw sequencing files were aligned using the STAR alignment suite (version 2.3.0) (Dobin et al. 2013). Uniquely mapping reads with no more than two mismatches were reported and used in this analysis. Sequences were aligned to a combined genome of $S$. pombe (version: ASM294v2) and S. cerevisiae (sacCer3=S288C_reference_genome_R64-1-1_20110203.tgz), and reads were separated by species-specific chromosome names. Bedgraph files were created by recording only the most $3^{\prime}$ base of each read. For calculating the number of reads within desired regions, custom scripts were used to count the amount of read $3^{\prime}$ ends within provided coordinates. The counts at each position in a bedgraph file were normalized based on the relative amount of reads aligning to the spike-in genome.

\section{Mappability tracks}

When determining the read density for genes or other transcription units, the length of the region was defined as the number of "mappable" bases within the unit. Uniquely mappable positions for 36-mer sequence alignments were determined by first computing all possible 36-mer sequences across the genome of interest and then aligning them back to the genome. By restricting the reported hits to perfect and unique alignments, we were able to generate mappability files that describe the mappable regions of the genome. In the case of samples receiving spiked-in libraries (i.e., $S$. pombe cultures spiked into $S$. cerevisiae), mappability tracks were created by taking all possible 36-mer sequences derived from the $S$. cerevisiae genome and aligning them to a species-combined genome. By aligning S. cerevisiae 36-mer sequences to a composite genome and reporting only unique hits, we are able to identify all 36-mer sequences that (1) uniquely align to the $S$.

\section{Genome Research}

www.genome.org 
cerevisiae genome and are thus not repeat elements, and (2) are distinguishable from the spiked-in genome.

\section{Gene sets}

For S. cerevisiae, the original (annotated) gene list consisted of all transcription units identified by Pelechano et al. (2013), considering only the longest major transcript isoform for each model. For S. pombe, a general transfer format (version: ASM294v1.16) file for gene annotations was downloaded from ftp://ftp.ensembl genomes.org/pub/fungi/release-16/gtf/schizosaccharomyces_pombe/. Gene models used in this study were filtered and refined based on the following criteria. Genes had to be nonoverlapping with any other annotated transcription units on the same strand. Gene transcription was defined as the normalized read depth across mappable bases within the gene body. A background read depth was estimated based on the read density within a set of intergenic regions. Genes were only considered active if their gene-body read density was greater than expected $(P<0.01)$ based on a Poisson distribution with a background sequencing rate. Inactive genes were omitted from downstream analysis. Moreover, only genes for which we were able to identify an observed TSS were used in our analysis-S. cerevisiae: $n=4398$ (84\% of not-overlapping transcription units) and $S$. pombe: $n=4674$ (80\% of not-overlapping transcription units). In order to avoid instances in which upstream transcription interferes with a gene's perceived transcription, we calculated a run-through index for each gene. The runthrough index was defined as the ratio of reads within the upstream window from -300 bases to TSS, relative to a downstream region +250 to +550 . Genes were only used if their upstream run-through index was less than 1 . Although the figures in this work are based on PRO-cap-based reannotation of early annotations (ASM294v1.16), we found no effect of using an updated set of annotations for $S$. pombe (ASM294v2.30) on our results and figures. Only 23 of the shared genes appear to have $5^{\prime}$ or $3^{\prime}$ coordinate changes. All genes for which we called an observed TSS are listed in Supplemental Table S2 (S. cerevisiae) and Supplemental Table S3 (S. pombe). In Supplemental Tables S2 and S3, normalized, background-subtracted PRO-cap signal for each gene is provided, and filtered genes used in this work are distinguished from unused genes.

\section{Observed TSS identification}

Our approach for calling transcription start sites with PRO-cap data is modeled after a similar analysis used for Start-seq data in mouse (Scruggs et al. 2015). Using the starting lists of TSS annotations, we developed an algorithm to search for the position within \pm 250 bases of each annotation possessing the highest PRO-cap read count on the corresponding strand. Importantly, we assess the background signal of our PRO-cap libraries with a TAP-minus replicate experiment. Thus, to identify the true most preferred base near the annotated TSS, we specifically looked for the position with the highest background-subtracted signal. In cases in which two or more bases shared the same signal intensity, the base closest to the annotation was chosen. Although situations arose in which multiple bases appeared to be used as TSSs, we restricted our analysis to the most preferred site to simplify downstream analysis. An observed TSS was required to have at least five reads more than the relative background signal.

\section{Pausing and termination index analysis}

Pausing index was quantified as the read density per mappable base within the first $100 \mathrm{bp}$ downstream from the observed TSS divided by the read density per mappable base within the gene body
(TSS +200 to CPS). The termination index was calculated by identifying the 100 -bp window 0 to $+500 \mathrm{bp}$ downstream from the CPS with the maximum read density per mappable base and dividing by the read density per mappable base within the gene body. For determining the number of paused genes within each WT yeast strain, we used the combined data from biological replicates to calculate pausing index. We further tested the significance of pausing within each gene by using Fisher's exact test to assess the likelihood of obtaining each ratio by chance. Using Bonferroni's correction, Fisher's exact test $P$-values were adjusted based on the number of genes analyzed. We called a gene paused if it had an adjusted $P$-value $<0.01$ and not paused if $P>0.99$. For calling highconfidence paused and not paused genes, we further restricted the respective gene sets based on analysis of individual biological replicates. For both biological replicates, a gene was required to have an adjusted $P$-value $<0.05$ (high-confidence paused) or $P>0.095$ (high-confidence not paused).

\section{MNase data}

Aligned and processed MNase-seq files were downloaded from GEO (S. pombe accession: GSE49575; S. cerevisiae accession: GSM1143089) for use in our analysis (DeGennaro et al. 2013; Hu et al. 2014). Nucleosome centers were previously identified by independent groups, i.e., S. pombe (Givens et al. 2012) and S. cerevisiae (Weiner et al. 2010). To ensure that the nucleosome centers used accurately reflected the distinct MNase-seq data, we assessed average MNase profiles relative to the centers. We found that the MNase-seq profiles of data used in this work corresponded nearly perfectly (within $\sim 5$ bp from MNase peaks on average) to the lists of nucleosome centers.

\section{Data access}

The raw and processed sequencing files from this study have been submitted to the NCBI Gene Expression Omnibus (GEO; http:// www.ncbi.nlm.nih.gov/geo/) under accession number GSE76142.

\section{Acknowledgments}

Research reported in this publication was supported by the National Institutes of Health (R01GM25232). The content is solely the responsibility of the authors and does not necessarily represent the official views of the National Institutes of Health. We thank Cornell Genomics Facility for overseeing the sequencing of our libraries.

Author contributions: G.T.B. conducted the experiments and data analysis; G.T.B. and J.T.L. conceived the experiments and analysis and wrote the manuscript; and V.G.C. and I.X.W. devised early PRO-seq adaptations for yeast and generated preliminary data in the strains used.

\section{References}

Adelman K, Lis JT. 2012. Promoter-proximal pausing of RNA polymerase II: emerging roles in metazoans. Nat Rev Genet 13: 720-731.

Brannan K, Kim H, Erickson B, Glover-Cutter K, Kim S, Fong N, Kiemele L, Hansen K, Davis R, Lykke-Andersen J, et al. 2012. mRNA decapping factors and the exonuclease Xrn2 function in widespread premature termination of RNA polymerase II transcription. Mol Cell 46: 311-324.

Castel SE, Ren J, Bhattacharjee S, Chang AY, Sánchez M, Valbuena A, Antequera F, Martienssen RA. 2014. Dicer promotes transcription termination at sites of replication stress to maintain genome stability. Cell 159: $572-583$.

Churchman LS, Weissman JS. 2011. Nascent transcript sequencing visualizes transcription at nucleotide resolution. Nature 469: 368-373. 
Collart MA, Oliviero S. 2001. Preparation of yeast RNA. Curr Protoc Mol Biol. doi: 10.1002/0471142727.mb1312s23.

Connelly S, Manley JL. 1988. A functional mRNA polyadenylation signal is required for transcription termination by RNA polymerase II. Genes Dev 2: $440-452$.

Core LJ, Waterfall JJ, Lis JT. 2008. Nascent RNA sequencing reveals widespread pausing and divergent initiation at human promoters. Science 322: $1845-1848$.

Core LJ, Waterfall JJ, Gilchrist DA, Fargo DC, Kwak H, Adelman K, Lis JT. 2012. Defining the status of RNA polymerase at promoters. Cell Rep 2: 1025-1035

Coudreuse D, van Bakel H, Dewez M, Soutourina J, Parnell T, Vandenhaute J, Cairns B, Werner M, Hermand D. 2010. A gene-specific requirement of RNA polymerase II CTD phosphorylation for sexual differentiation in $S$. pombe. Curr Biol 20: 1053-1064.

Crooks GE, Hon G, Chandonia JM, Brenner SE. 2004. WebLogo: a sequence logo generator. Genome Res 14: 1188-1190.

Danko CG, Hah N, Luo X, Martins AL, Core L, Lis JT, Siepel A, Kraus WL. 2013. Signaling pathways differentially affect RNA polymerase II initiation, pausing, and elongation rate in cells. Mol Cell 50: 212-222.

DeGennaro CM, Alver BH, Marguerat S, Stepanova E, Davis CP, Bähler J, Park PJ, Winston F. 2013. Spt6 regulates intragenic and antisense transcription, nucleosome positioning, and histone modifications genome-wide in fission yeast. Mol Cell Biol 33: 4779-4792.

Dobin A, Davis CA, Schlesinger F, Drenkow J, Zaleski C, Jha S, Batut P, Chaisson M, Gingeras TR. 2013. STAR: ultrafast universal RNA-seq aligner. Bioinformatics 29: 15-21.

Dujon B. 2010. Yeast evolutionary genomics. Nat Rev Genet 11: 512-524.

Eser P, Wachutka L, Maier KC, Demel C, Boroni M, Iyer S, Cramer P, Gagneur J. 2016. Determinants of RNA metabolism in the Schizosaccharomyces pombe genome. Mol Syst Biol 12: 857.

Fong N, Brannan K, Erickson B, Kim H, Cortazar MA. 2015. Effects of transcription elongation rate and Xrn2 exonuclease activity on RNA polymerase II termination suggest widespread kinetic competition. Mol Cell 60: $256-267$

Fuda NJ, Ardehali MB, Lis JT. 2009. Defining mechanisms that regulate RNA polymerase II transcription in vivo. Nature 461: 186-192.

García-Martínez J, Aranda A, Pérez-Ortín JE. 2004. Genomic run-on evaluates transcription rates for all yeast genes and identifies gene regulatory mechanisms. Mol Cell 15: 303-313.

Gilchrist DA, Fromm G, dos Santos G, Pham LN, McDaniel IE, Burkholder A, Fargo DC, Adelman K. 2012. Regulating the regulators: the pervasive effects of Pol II pausing on stimulus-responsive gene networks. Genes Dev 26: 933-944.

Givens RM, Lai WK, Rizzo JM, Bard JE, Mieczkowski PA, Leatherwood J, Huberman JA, Buck MJ. 2012. Chromatin architectures at fission yeast transcriptional promoters and replication origins. Nucleic Acids Res 40: 7176-7189.

Gromak N, West S, Proudfoot NJ. 2006. Pause sites promote transcriptional termination of mammalian RNA polymerase II. Mol Cell Biol 26: 3986-3996.

Hahn S, Young ET. 2011. Transcriptional regulation in Saccharomyces cerevisiae: transcription factor regulation and function, mechanisms of initiation, and roles of activators and coactivators. Genetics 189: 705-736.

Hall MA, Shundrovsky A, Bai L, Fulbright RM, Lis JT, Wang MD. 2009. Highresolution dynamic mapping of histone-DNA interactions in a nucleosome. Nat Struct Mol Biol 16: 124-129.

Hartzog GA, Fu J. 2013. The Spt4-Spt5 complex: a multi-faceted regulator of transcription elongation. Biochim Biophys Acta 1829: 105-115.

Hu Z, Chen K, Xia Z, Chavez M, Pal S, Seol JH, Chen CC, Li W, Tyler JK. 2014. Nucleosome loss leads to global transcriptional up-regulation and genomic instability during yeast aging. Genes Dev 28: 396-408.

Jiang C, Pugh BF. 2009. Nucleosome positioning and gene regulation: advances through genomics. Nat Rev Genet 10: 161-172.

Jonkers I, Kwak H, Lis JT. 2014. Genome-wide dynamics of Pol II elongation and its interplay with promoter proximal pausing, chromatin, and exons. eLife 3: e02407.

Jordán-Pla A, Gupta I, de Miguel-Jiménez L, Steinmetz LM, Chávez S, Pelechano V, Pérez-Ortín JE. 2014. Chromatin-dependent regulation of RNA polymerases II and III activity throughout the transcription cycle. Nucleic Acids Res 43: 787-802.

Keogh MC, Podolny V, Buratowski S. 2003. Bur1 kinase is required for efficient transcription elongation by RNA polymerase II. Mol Cell Biol 23: 7005-7018.

Kruesi WS, Core LJ, Waters CT, Lis JT, Meyer BJ. 2013. Condensin controls recruitment of RNA polymerase II to achieve nematode X-chromosome dosage compensation. eLife 2: e00808.

Kwak H, Fuda NJ, Core LJ, Lis JT. 2013. Precise maps of RNA polymerase reveal how promoters direct initiation and pausing. Science 339: 950-953.
Laitem C, Zaborowska J, Isa NF, Kufs J, Dienstbier M, Murphy S. 2015. CDK9 inhibitors define elongation checkpoints at both ends of RNA polymerase II-transcribed genes. Nat Struct Mol Biol 22: 396-403.

Langmead B, Trapnell C, Pop M, Salzberg SL. 2009. Ultrafast and memoryefficient alignment of short DNA sequences to the human genome. Genome Biol 10: R25.

Li J, Liu Y, Rhee HS, Ghosh SKB, Bai L, Pugh BF, Gilmour DS. 2013. Kinetic competition between elongation rate and binding of NELF controls promoter-proximal pausing. Mol Cell 50: 711-722.

Li H, Hou J, Bai L, Hu C, Tong P, Kang Y, Zhao X, Shao Z. 2015. Genomewide analysis of core promoter structures in Schizosaccharomyces pombe with DeepCAGE. RNA Biol 12: 525-537.

Love JD, Vivino AA, Minton KW. 1985. Detection of low-level gene induction using in vitro transcription heat shock genes. Gene Anal Tech 2: 100-107.

Love MI, Huber W, Anders S. 2014. Moderated estimation of fold change and dispersion for RNA-seq data with DESeq2. Genome Biol 15: 550 .

Mason PB, Struhl K. 2005. Distinction and relationship between elongation rate and processivity of RNA polymerase II in vivo. Mol Cell 17: 831-840.

Maxwell CS, Kruesi WS, Core LJ, Kurhanewicz N, Waters CT, Lewarch CL, Antoshechkin I, Lis JT, Meyer BJ, Baugh LR. 2014. Pol II docking and pausing at growth and stress genes in C. elegans. Cell Rep 6: 455-466.

Mayer A, Lidschreiber M, Siebert M, Leike K, Söding J, Cramer P. 2010. Uniform transitions of the general RNA polymerase II transcription complex. Nat Struct Mol Biol 17: 1272-1278.

McKinlay A, Araya CL, Fields S. 2011. Genome-wide analysis of nascent transcription in Saccharomyces cerevisiae. G3 (Bethesda) 1: 549-558.

Min IM, Waterfall JJ, Core LJ, Munroe RJ, Schimenti J, Lis JT. 2011. Regulating RNA polymerase pausing and transcription elongation in embryonic stem cells. Genes Dev 25: 742-754.

Narita T, Yamaguchi Y, Yano K, Sugimoto S, Chanarat S, Wada T, Kim DK, Hasegawa J, Omori M, Inukai N, et al. 2003. Human transcription elongation factor NELF: identification of novel subunits and reconstitution of the functionally active complex. Mol Cell Biol 23: 1863-1873.

Neil H, Malabat C, d'Aubenton-Carafa Y, Xu Z, Steinmetz LM, Jacquier A. 2009. Widespread bidirectional promoters are the major source of cryptic transcripts in yeast. Nature 457: 1038-1042.

Pei Y, Shuman S. 2002. Interactions between fission yeast mRNA capping enzymes and elongation factor Spt5. J Biol Chem 277: 19639-19648.

Pei Y, Schwer B, Shuman S. 2003. Interactions between fission yeast Cdk9, its cyclin partner Pch1, and mRNA capping enzyme Pct1 suggest an elongation checkpoint for mRNA quality control. J Biol Chem 278: 7180-7188.

Pei Y, Du H, Singer J, Stamour C, Granitto S, Shuman S, Fisher RP. 2006. Cyclin-dependent kinase 9 (Cdk9) of fission yeast is activated by the CDK-activating kinase Csk1, overlaps functionally with the TFIIH-associated kinase Mcs6, and associates with the mRNA cap methyltransferase Pcm1 in vivo. Mol Cell Biol 26: 777-788.

Pelechano V, Steinmetz LM. 2013. Gene regulation by antisense transcription. Nat Rev Genet 14: 880-893.

Pelechano V, Chávez S, Pérez-Ortín JE. 2010. A complete set of nascent transcription rates for yeast genes. PLoS One 5: e15442.

Pelechano V, Wei W, Steinmetz LM. 2013. Extensive transcriptional heterogeneity revealed by isoform profiling. Nature 497: 127-131.

Peterlin BM, Price DH. 2006. Controlling the elongation phase of transcription with P-TEFb. Mol Cell 23: 297-305.

Ping YH. 2000. DSIF and NELF interact with RNA polymerase II elongation complex and HIV-1 Tat stimulates P-TEFb-mediated phosphorylation of RNA polymerase II and DSIF during transcription elongation. $J$ Biol Chem 276: 12951-12958.

Porrua O, Libri D. 2015. Transcription termination and the control of the transcriptome: why, where and how to stop. Nat Rev Mol Cell Biol 16: 190-202.

Proudfoot NJ. 1989. How RNA polymerase II terminates transcription in higher eukaryotes. Trends Biochem Sci 14: 105-110.

Ptashne M, Gann A. 1997. Transcriptional activation by recruitment. Nature 386: $569-577$.

Rahl PB, Lin CY, Seila AC, Flynn RA, McCuine S. 2010. c-Myc regulates transcriptional pause release. Cell 141: 432-445.

Rhee HS, Pugh BF. 2012. Genome-wide structure and organization of eukaryotic pre-initiation complexes. Nature 483: 295-301.

Rodríguez-Gil A, García-Martínez J, Pelechano V, Muñoz-Centeno Mde LC, Geli V, Pérez-Ortín JE, Chávez S. 2010. The distribution of active RNA polymerase II along the transcribed region is gene-specific and controlled by elongation factors. Nucleic Acids Res 38: 4651-4664.

Rondón AG, García-Rubio M, González-Barrera S, Aguilera A. 2003 Molecular evidence for a positive role of Spt4 in transcription elongation. EMBO J 22: 612-620.

Sansó M, Lee KM, Viladevall L, Jacques PÉ, Pagé V, Nagy S, Racine A, St Amour CV, Zhang C, Shokat KM, et al. 2012. A positive feedback loop links opposing functions of $\mathrm{P}-\mathrm{TEFb} / \mathrm{Cdk9}$ and histone $\mathrm{H} 2 \mathrm{~B}$ 
ubiquitylation to regulate transcript elongation in fission yeast. PLoS Genet 8: e1002822.

Schneider S, Pei Y, Shuman S, Schwer B. 2010. Separable functions of the fission yeast Spt5 carboxyl-terminal domain (CTD) in capping enzyme binding and transcription elongation overlap with those of the RNA polymerase II CTD. Mol Cell Biol 30: 2353-2364.

Schwer B, Schneider S, Pei Y, Aronova A, Shuman S. 2009. Characterization of the Schizosaccharomyces pombe Spt5-Spt4 complex. RNA 15: 1241-1250.

Scruggs BS, Gilchrist DA, Nechaev S, Muse GW. 2015. Bidirectional transcription arises from two distinct hubs of transcription factor binding and active chromatin. Mol Cell 58: 1101-1112.

Stargell LA, Struhl K. 1996. Mechanisms of transcriptional activation in vivo: two steps forward. Trends Genet 12: 311-315.

Steinmetz EJ, Warren CL, Kuehner JN, Panbehi B, Ansari AZ, Brow DA. 2006. Genome-wide distribution of yeast RNA polymerase II and its control by Sen1 helicase. Mol Cell 24: 735-746.

Struhl K, Segal E. 2013. Determinants of nucleosome positioning. Nat Struct Mol Biol 20: 267-273.

Sun M, Schwalb B, Schulz D, Pirkl N, Etzold S, Larivière L, Maier KC, Seizl M, Tresch A, Cramer P. 2012. Comparative dynamic transcriptome analysis (cDTA) reveals mutual feedback between mRNA synthesis and degradation. Genome Res 22: 1350-1359.

Swanson MS, Malone EA, Winston F. 1991. SPT5, an essential gene important for normal transcription in Saccharomyces cerevisiae, encodes an acidic nuclear protein with a carboxy-terminal repeat. Mol Cell Biol 11: $3009-3019$.

Thomas PD, Campbell MJ, Kejariwal A, Mi H, Karlak B, Daverman R, Diemer K, Muruganujan A, Narechania A. 2003. PANTHER: a library of protein families and subfamilies indexed by function. Genome Res 13: 2129-2141.

Venkatesh S, Workman JL. 2015. Histone exchange, chromatin structure and the regulation of transcription. Nat Rev Mol Cell Biol 16: 178-189.
Viladevall L, St Amour CV, Rosebrock A, Schneider S, Zhang C, Allen JJ, Shokat KM, Schwer B, Leatherwood JK, Fisher RP. 2009. TFIIH and P$\mathrm{TEFb}$ coordinate transcription with capping enzyme recruitment at specific genes in fission yeast. Mol Cell 33: 738-751.

Wada T. 1998. Evidence that P-TEFb alleviates the negative effect of DSIF on RNA polymerase II-dependent transcription in vitro. EMBO J 17: 7395-7403.

Wada T, Takagi T, Yamaguchi Y, Ferdous A, Imai T, Hirose S, Sugimoto S, Yano K, Hartzog GA, Winston F, et al. 1998. DSIF, a novel transcription elongation factor that regulates RNA polymerase II processivity, is composed of human Spt4 and Spt5 homologs. Genes Dev 12: 343-356.

Weber CM, Ramachandran S, Henikoff S. 2014. Nucleosomes are contextspecific, H2A.Z-modulated barriers to RNA polymerase. Mol Cell 53: 819-830.

Weiner A, Hughes A, Yassour M, Rando OJ, Friedman N. 2010. High-resolution nucleosome mapping reveals transcription-dependent promoter packaging. Genome Res 20: 90-100.

Wilhelm BT, Marguerat S, Watt S, Schubert F, Wood V, Goodhead I, Penkett CJ, Rogers J, Bähler J. 2008. Dynamic repertoire of a eukaryotic transcriptome surveyed at single-nucleotide resolution. Nature 453: 1239-1243.

Wu CH, Yamaguchi Y, Benjamin LR, Horvat-Gordon M, Washinsky J, Enerly E, Larsson J, Lambertsson A, Handa H, Gilmour D. 2003. NELF and DSIF cause promoter proximal pausing on the $h s p 70$ promoter in Drosophila. Genes Dev 17: 1402-1414.

Xu Z, Wei W, Gagneur J, Perocchi F, Clauder-Münster S, Camblong J, Guffanti E, Stutz F, Huber W, Steinmetz LM. 2009. Bidirectional promoters generate pervasive transcription in yeast. Nature 457: 1033-1037.

Received January 18, 2016; accepted in revised form April 19, 2016. 


\section{Corrigendum}

Genome Research 26: 799-811 (2016)

\section{Corrigendum: Divergence of a conserved elongation factor and transcription regulation in budding and fission yeast}

Gregory T. Booth, Isabel X. Wang, Vivian G. Cheung, and John T. Lis

Figure 1C and Supplemental Figure S1C in the above article displayed incorrect sequence logos based on the observed TSS. Observed positions on the plus strand were shifted by one base, causing a misalignment of underlying sequences. The authors would like to correct these panels and the text referring to them on pages 800801 ("Results," first subsection, second paragraph), which should read as follows:

"Moreover, a moderate sequence preference for initiating at an A/G, immediately downstream from a C/T, was revealed under the observed TSS, whereas no base preferences underlie the PomBase annotations for the same genes (Fig. 1C)."

The corrected Figure 1 is provided on the next page.

Supplemental Tables S2 and S3 have also been corrected to remove this shift.

The authors thank Craig Kaplan for bringing this to their attention, and apologize for any confusion this may have caused.

The article has already been corrected in both the PDF and full-text HTML files online.

doi: 10.1101/gr.210161.116 
A

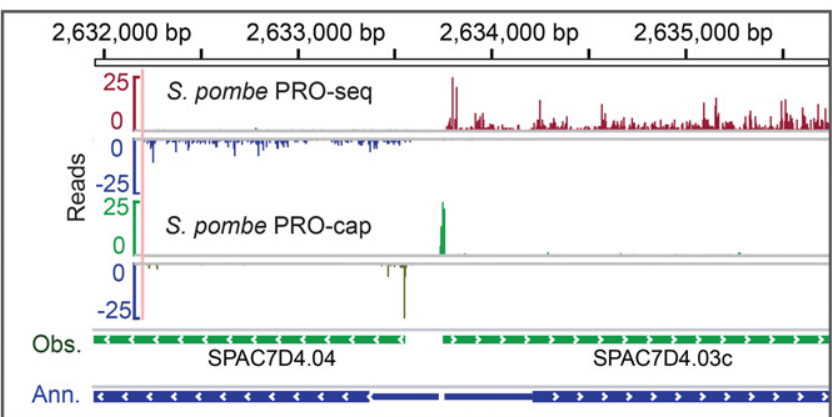

B

S. pombe Annotated TSS

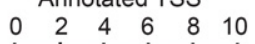
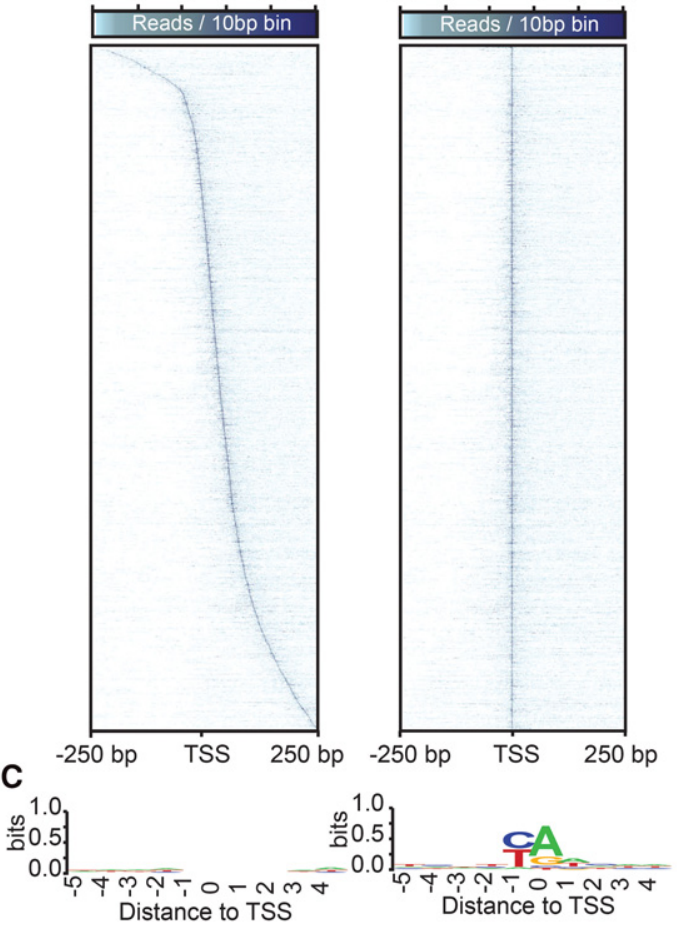

D

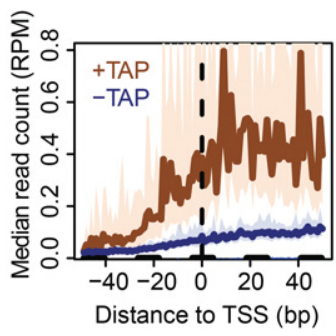

E

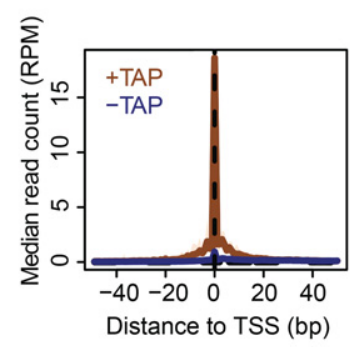

$\mathbf{F}$

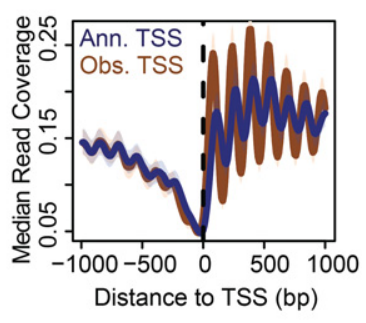

Figure 1. 


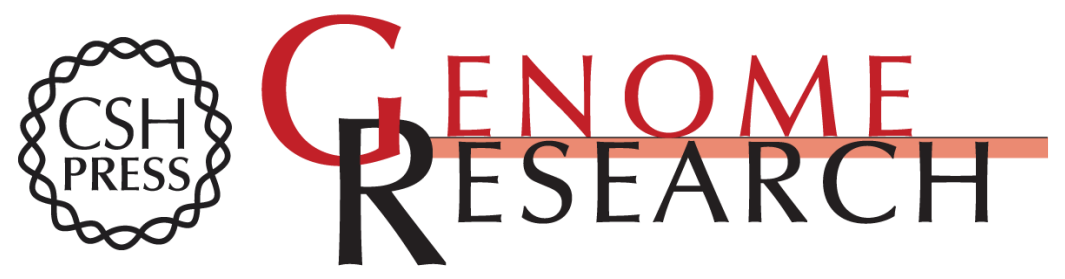

\section{Divergence of a conserved elongation factor and transcription regulation in budding and fission yeast}

Gregory T. Booth, Isabel X. Wang, Vivian G. Cheung, et al.

Genome Res. 2016 26: 799-811 originally published online May 12, 2016

Access the most recent version at doi:10.1101/gr.204578.116

\section{Supplemental Material \\ Related Content \\ References Commons License}

Email Alerting Service
http://genome.cshlp.org/content/suppl/2016/05/12/gr.204578.116.DC1

http://genome.cshlp.org/content/suppl/2016/05/24/gr.204578.116.DC2

Corrigendum: Divergence of a conserved elongation factor and transcription regulation in budding and fission yeast Gregory T. Booth, Isabel X. Wang, Vivian G. Cheung, et al.

Genome Res. July , 2016 26: 1010-1011

This article cites 78 articles, 27 of which can be accessed free at:

http://genome.cshlp.org/content/26/6/799.full.html\#ref-list-1

Articles cited in:

http://genome.cshlp.org/content/26/6/799.full.html\#related-urls

Creative This article is distributed exclusively by Cold Spring Harbor Laboratory Press for the first six months after the full-issue publication date (see http://genome.cshlp.org/site/misc/terms.xhtml). After six months, it is available under a Creative Commons License (Attribution-NonCommercial 4.0 International), as described at http://creativecommons.org/licenses/by-nc/4.0/.

Receive free email alerts when new articles cite this article - sign up in the box at the top right corner of the article or click here.

\section{Affordable, Accurate Sequencing.}

To subscribe to Genome Research go to: https://genome.cshlp.org/subscriptions 